\title{
Effect of a chemical inhibitor of human phosphatidylethanolamine-binding protein 4 on radiosensitivity of rectal cancer cells
}

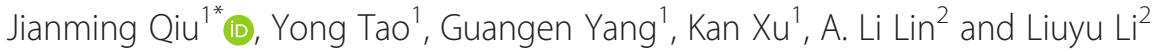

\begin{abstract}
Background: Human phosphatidylethanolamine-binding protein 4 (hPEBP4) is a well-established antiapoptosis molecule in recent years. It has also been demonstrated to be involved in the radioresistance of rectal cancer. The objective of this study was to determine whether IOI-42, a chemical inhibitor of hPEBP4, could sensitize rectal cancer cells.

Methods: Rectal cancer cells were treated with IOI-42 alone or in combination with irradiation. Clonogenic survival assays and tumor volume growth analysis were used, respectively, to study the effect of IOI-42 in vitro and in vivo. Western blot was adopted to measure the activation of signal pathway.

Results: Clonogenic survival assays showed that IOI-42, combined with irradiation, caused a significant decrease in colony formation compared with radiation alone, which was associated with the downregulation of Akt activation. And we also confirmed the effect of $\mathrm{IOI}-42$ in nude mice transplanted with human rectal cancer subcutaneously.

Conclusions: These data suggest that IOI-42 has a potential to enhance the radiosensitivity of rectal cancer cells, providing a rationale to further investigate the feasibility of combining of $\mathrm{IOI}-42$ with radiation, keeping in mind that this may result in unexpected toxicities.
\end{abstract}

Keywords: Human phosphatidylethanolamine-binding protein 4, Radiosensitivity, Rectal cancer

Abbreviation: hPEBP4, Human phosphatidylethanolamine-binding protein 4

\section{Background}

The phosphatidylethanolamine-binding protein (PEBP) family consists of a number of $21-23-\mathrm{kDa}$ basic proteins, first identified in the bovine brain, with preferential in vitro affinity for phosphatidylethanolamine, a component of the cell membrane [1]. A number of functions have been suggested for the mammalian PEBP proteins, including lipid binding and inhibition of serine proteases [2]. Human phosphatidylethanolamine-binding protein 4 (hPEBP4) is a novel member of the PEBP family [3]. A recent study shows that hPEBP4 was an independent predictive biomarker for the response of rectal cancer to preoperative radiotherapy [4]. The radioresistance effect of hPEBP4 has been demonstrated to be associated with

\footnotetext{
* Correspondence: 15168427344@163.com

'Department of Colorectal Surgery, The Third Hospital of Hangzhou, 38 West Lake Avenue, ShangChen District, Hangzhou, China

Full list of author information is available at the end of the article
}

Akt activation [5]. At the very beginning, the study on hPEBP4 has focused on its antiapoptosis effect [4, 6-9]. A specific chemical inhibitor of hPEBP4 was also found through virtual screening, which showed significant antiapoptosis effect by targeting the conservative PE-binding domain of the molecule of hPEBP4 [10]. Thus, we speculated that this inhibitor named as IOI-42 may also enhance the radiosensitivity of rectal cancer. In this study, we evaluated the radiosensitizing effect of IOI- 42 both in vitro and in vivo using the model of rectal cancer cells and nude mice transplanted with human rectal tumor, respectively.

\section{Methods \\ Antibody and cells}

Antibodies specific to phospho-Akt (Ser473) were from Cell Signaling Technology (Beverly, MA). LY-294002 was purchased from Sigma-Aldrich (Shanghai, China). All human rectal cancer cells including HRT18 and HT- 
29 were purchased from the American Type Culture Collection (Manassas, VA) and maintained in a DMEM medium (Gibco, USA) supplemented with $10 \%(v / v)$ fetal calf serum, $4.5 \mathrm{~g} / \mathrm{l} \mathrm{D}$-glucose, nonessential amino acids (100 mM each), 100 units $/ \mathrm{ml}$ penicillin, $100 \mathrm{mg} /$ $\mathrm{ml}$ streptomycin, and $2 \mathrm{mM}$ glutamine at $37{ }^{\circ} \mathrm{C}$ in a $5 \%$ $\mathrm{CO}_{2}$ atmosphere.

\section{Chemical inhibitor of hPEBP4}

The structure of IOI-42 was identical as that shown in the original study [8]. A $10-\mathrm{mM}$ stock solution was prepared in sterile $0.9 \%$ sodium chloride and stored at $4{ }^{\circ} \mathrm{C}$. In this study, IOI-42 was used at increasing concentrations from 1 to $10 \mu \mathrm{M}$.

\section{Clonogenic survival assay}

Exponentially growing rectal cancer cells in monolayer culture were irradiated in 100-mm Petri dishes using a 250-kVp X-ray $(0.61 \mathrm{~Gy} / \mathrm{min})$ by single radiation exposure. After exposure to ionizing irradiation, the cells were harvested for clonogenic survival analysis. Survival after radiation exposure was defined as the ability of the cells to maintain their clonogenic capacity and form colonies. Briefly, after exposure to radiation, cells were trypsinized, counted, and seeded for colony formation in 100$\mathrm{mm}$ Petri dishes at 500-1000 cells/dish. After incubation intervals of 21 days, colonies were stained with crystal violet and counted manually. Colonies consisting of more than 50 cells were scored, and three replicate dishes were counted for each treatment.

Experiments were carried out at least three times for all data points. The experimental results were corrected for effects induced by the nonirradiated control.

\section{In vivo radiation studies}

Male athymic nu/nu mice (4 weeks old) were purchased from the Experimental Animal Center of Shanghai and were raised using protocols that had been approved by the Institutional Animal Care and Use Committee. Forty-eight mice were randomly divided into four groups. The mice were injected subcutaneously with $2 \times$ $10^{6}$ HRT-18 cells on the right foot pad. When tumors were approximately $0.5-0.8 \mathrm{~cm}$ in the longest diameter, the mice of four groups were subject to no treatment, irradiation alone, irradiation with IOI-42 intratumoral injection, or IOI-42 alone. Irradiation regimen was as the following: 2 Gy Cs-radiation source every 3 days for three times as described before [11]. Tumors were monitored and measured with the formula $V=\left(a \times b^{2}\right) / 2$ every 3 days. Observation was closed once the average volume of tumor of any group reached $3.0 \mathrm{~cm}^{3}$. All mice were executed 33 days after radiation exposure, and tumor size-time curve as well as the apoptosis of tumor tissues was analyzed.

\section{Immunohistochemistry analysis of Akt activation and expression of DNA repair genes}

Transplanted human rectal cancer tissues were obtained from the executed mice. The specimens were fixed with formalin, embedded in paraffin, and immunostained with specific antibody using avidin-biotin peroxidase complex method. Immunoreactivity was evaluated based on the percent of positive-stained cells.

\section{TUNEL assay}

Transplanted tumors from mice were collected and fixed in $10 \%$ formalin and embedded in paraffin after mice were excised. After deparaffinization, the tumor samples were stripped of protein by incubation with $20 \mathrm{mg} / \mathrm{ml}$ proteinase K (Sigma Chemical) for $15 \mathrm{~min}$ at room temperature. TUNEL staining was performed using an apoptosis in situ detection kit (TaKaRa) according to the manufacture's protocols. The frequency of apoptosis was calculated as an apoptotic index, in which more than 200 positive cells were examined in five random fields of each group, and TUNEL-positive cells were counted to calculate the percent of positive cells as the apoptosis index in situ.

\section{Statistical analysis}

Data are expressed as mean values \pm SD. Statistical significance of differences between groups was tested by Student's $t$ test, one-way ANOVA, or chi-square test. A $p$ value of less than 0.05 was considered significant.

\section{Results}

Effects of IOI-42 on clonogenic survival of rectal cancer cells after irradiation

In order to evaluate the radiosensitizing effect of IOI-42 on rectal cancer in vitro, we examined the effect of IOI42 on the clonogenic survival of two rectal cancer cell lines combined with irradiation. We found that IOI-42 itself did not influence the survival of both HRT-18 and HT-29 cells. But it significantly enhanced the killing of rectal cancer cells by irradiation (Fig. 1a, b). Then, we investigated the concentration dependence inhibition of colony formation of these cell lines for different concentrations of IOI-42. As the concentration of IOI-42 increases, the survival of rectal cancer cells decreased after irradiation, and the higher the IOI- 42 concentration, the lower the survival of rectal cancer cells $(p<$ $0.05)$. However, when increased to a concentration of $10 \mu \mathrm{M}$, IOI-42 itself significantly reduced the survival of rectal cancer cells (Fig. 1c, d). So in the following experiments, we chose to study the radiosensitizing effect of IOI-42 at the concentration of $5 \mu \mathrm{M}$. 


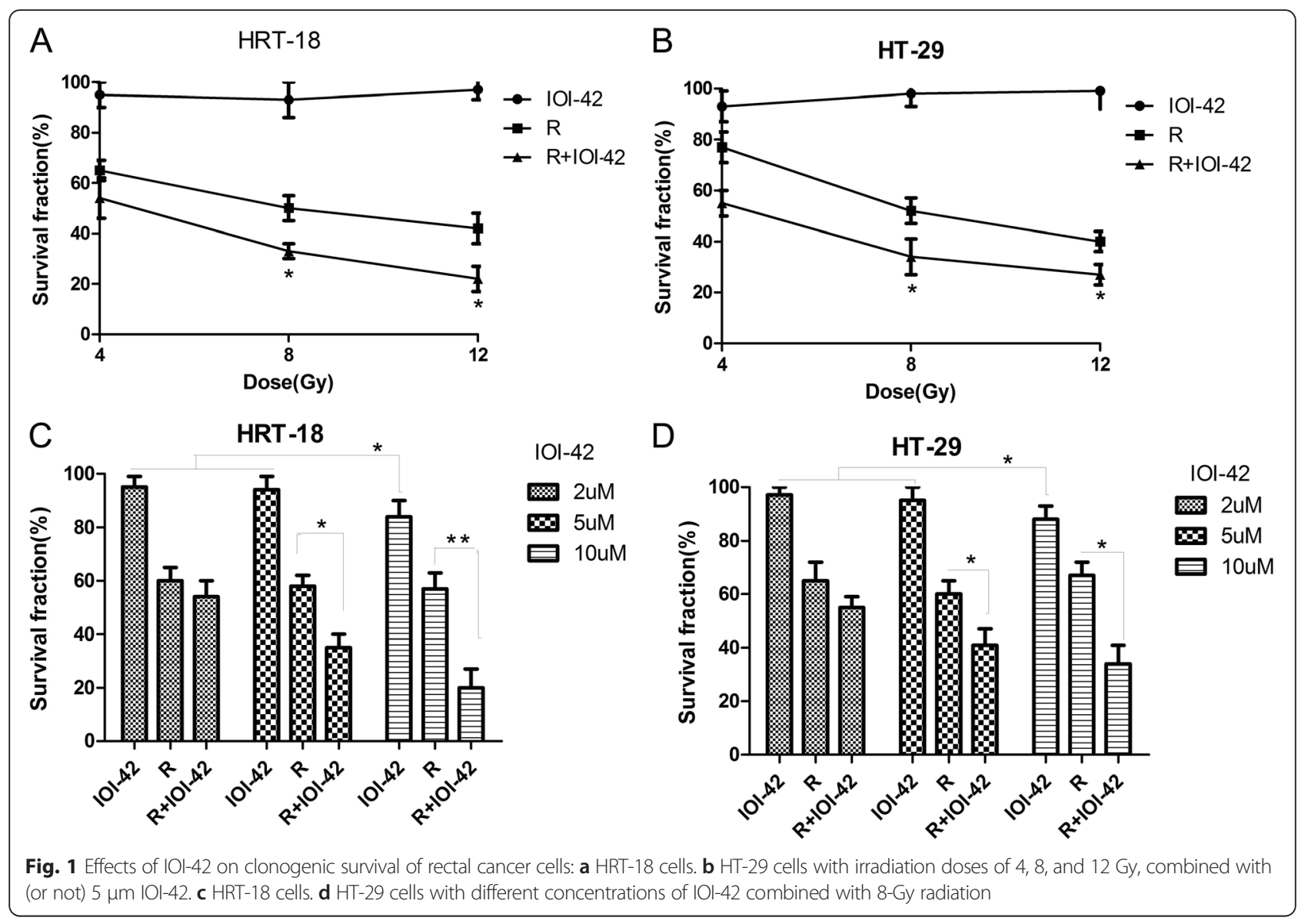

IOI-42-mediated radiosensitivity of rectal cancer cells was Akt dependent

Akt activation has been demonstrated to be essential in the hPEBP4-mediated radioresistance of rectal cancer [5], so we explored the role of Akt in the effect of IOI42. Firstly, we confirmed the activation of Akt after irradiation, its dramatic inhibition by $\mathrm{IOI}-42$, and its almost complete depression by pre-incubation with $20 \mu \mathrm{M}$ LY-294002 as well (Fig. 2a). Then, we examined the role of Akt activation in the radiosensitizing effect of IOI-42. We found that the effect of IOI-42 was totally abolished after LY-294002 inhibited activation of Akt for both HRT-18 and HT-29 cells (Fig. 2b).

IOI-42 promoted the sensitivity of rectal cancers to irradiation in vivo

To determine whether IOI-42 can also promote the radiosensitivity of colorectal cancer in vivo, we examined the effect of radiation alone, IOI-42 alone, or in combination on the growth of subcutaneous HT-29 xenograft rectal tumors in nude mice (Fig. 3a). We found that from the 12th day, the tumor volume in the combined treatment group was significantly smaller than that in the radiation only group $(p<0.05)$. And no growth delay

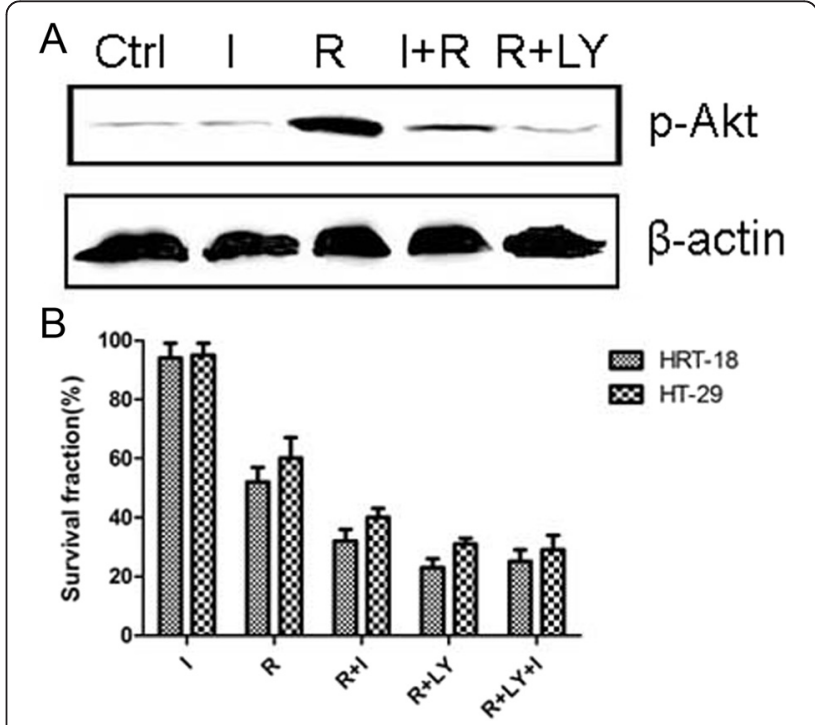

Fig. 2 IOl-42-mediated radiosensitivity of rectal cancer cells was Akt dependent. a Effect of radiation, IOI-42, and LY-294002 on the activation of Akt. b LY-294002 abolish the effect of IOI-42 both in HRT-18 and HT29 cells. (/ is the short form for IOI-42, $R$ is the short form for irradiation) 


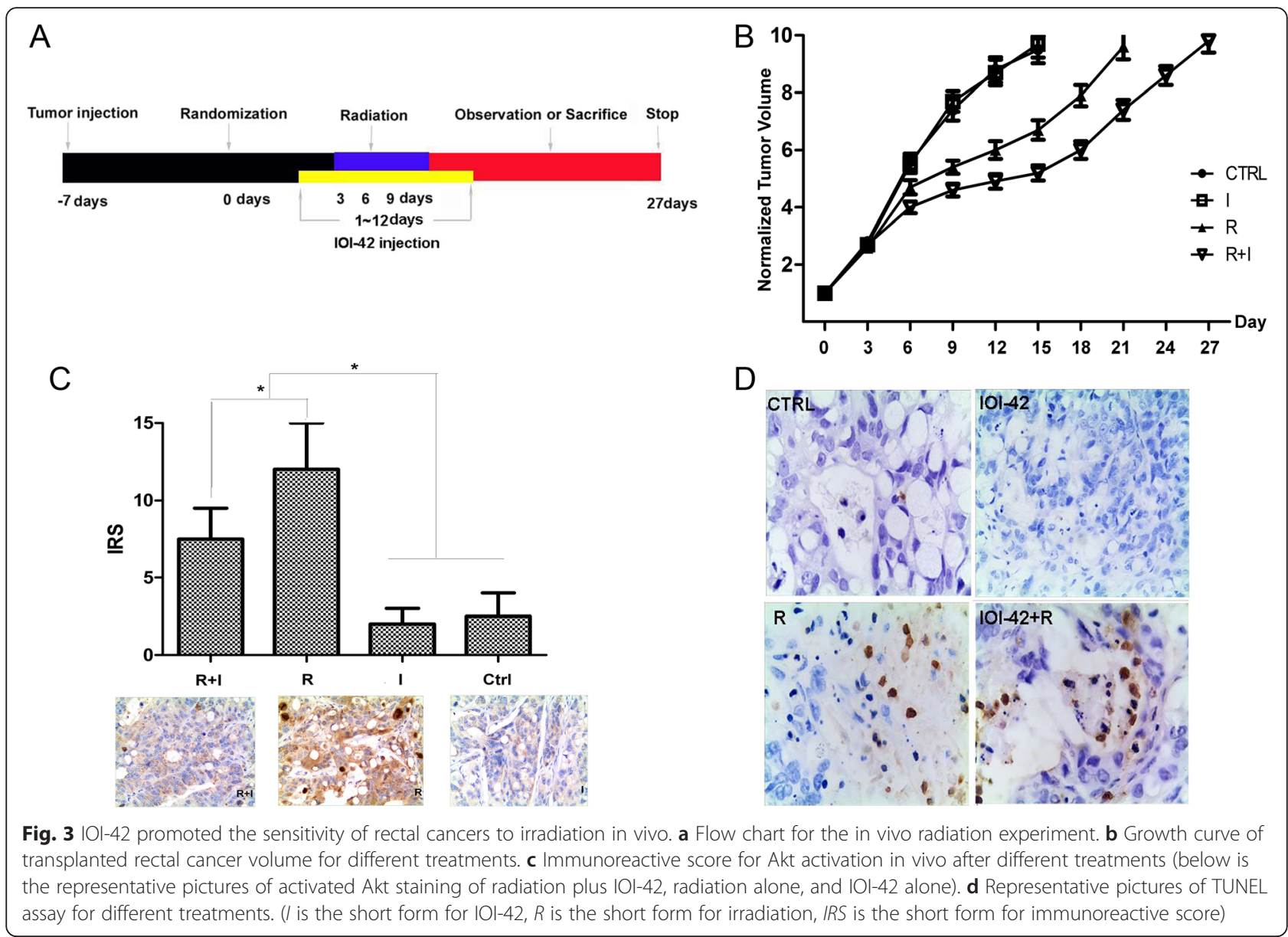

was observed in the IOI-42 only group compared with control (Fig. 3b). We also examined Akt activation of the tumor tissue with immunohistochemistry, which shows that IOI-42 significantly compromised irradiation-induced Akt activation (Fig. 3c). The effect of IOI-42 on the rectal tumor growth after irradiation was also confirmed by apoptosis stain in situ (Fig. 3d); the apoptosis index of the combined group is significantly higher than that of the group of radiation alone (1.5 vs $0.85, p<0.05)$.

\section{Discussion}

As the first chemical inhibitor of hPEBP4, IOI-42 has been demonstrated to be able to block the conservative PE-binding domain of hPEBP4 and reverse the signal pathway affected by hPEBP4 overexpression [10]. In the present study, we proved that IOI-42 could enhance the radiosensitivity of rectal cancer cells both in vitro and in vivo through inhibiting hPEBP4-induced Akt activation after irradiation.

Since hPEBP4 has been shown to be overexpressed in breast, prostate, and ovarian cancers [3, 6-9], our study suggested that IOI-42 might also be a potential radiosensitizing agent for all the involved human cancers. There have been seldom breakthrough in the development of radiosensitizing agents in recent years. To speed up the development of radiosensitizing agents, taking advantage of the differentially expressed gene profile of cancer rather than just focusing on some classical death signal pathway might be essential [12-15].

Consistent with previous study with siRNA to silence hPEBP4 [3], our study confirmed that inhibition of Akt activation is pivotal in the radiosensitizing effect of IOI-42. The upregulation of Akt activation by hPEBP4 was believed to be reactive oxygen species (ROS)-dependent, though we did not know the exact signal event downward of ROS, through which hPEBP4 activated Akt to promote the radioresistance of rectal cancer [5, 7]. Neither we know the final effect molecule after Akt activation. One thing is for sure that targeting the conservative PEbinding domain of the molecule of hPEBP4 is essential for IOI-42 in playing its radiosensitizing effect. To address that problem, we actually compared the expression of some nucleotide repair genes between irradiation alone and combination of irradiation with IOI-42 in this study but found no significant difference for nucleotide repair genes like FANCG, ERCC1, PMS1/2, BRCA1/2, LIG4, and 
TP53 [16-20]. So the detailed mechanism of hPEBP4induced radioresistance needs further exploration, which will promote the development of more chemical inhibitors of hPEBP4 and also the potential application of multitargeting chemicals with stronger radiosensitizing effect. Being a preliminary study of IOI-42 as a radiosensitizing agent for rectal cancer, we did not examine the side effect of IOI-42. But we did observe anorexia in some mice subject to IOI-42 when compared with control. It requires systematic research to clarify its potential toxic effect if IOI-42 turned out to be a promising agent to enhance radiosensitivity.

\section{Conclusions}

In summary, this is the very first report on a chemical inhibitor of hPEBP4 in promoting the radiosensitivity of rectal cancer, suggesting IOI-42 may be a potential radiation-sensitive agent in the clinical treatment of cancers. Further work is necessary to demonstrate its efficacy on other human cancers and also to evaluate its possible side effect.

\section{Acknowledgements}

We thank Dr Richard Sim for his useful review and Miss Huan Yu for her support regarding the work schedule.

\section{Funding}

This work was financially supported by the Natural Science Foundation of Zhejiang Province (project number LY15H160001; LY15H030001) and the Medical Fund for Special Diseases of Key Department of Hangzhou (project number 20150733Q28) ranging from the design of the study, collection, analysis, and interpretation of data to the writing the manuscript.

\section{Availability of data and materials}

The datasets generated during and/or analyzed during the current study are not publicly available due to the privacy of drug development but are available from the corresponding author on reasonable request.

\section{Authors' contributions}

JQ came up with the hypothesis, designed the whole set of experiments, and organized the group to finish the work. YT carried out most of the experiments and collected and analyzed the data. GY gave good advice on the in vivo radiation experiments. $\mathrm{KX}$ helped to make the transplanted rectal tumor in the mice. A-LL and LL did all the histological analyses. All authors read and approved the final manuscript.

\section{Competing interests}

The authors declare that they have no competing interests.

\section{Consent for publication}

Not applicable.

\section{Ethics approval and consent to participate}

Not applicable.

\section{Author details}

'Department of Colorectal Surgery, The Third Hospital of Hangzhou, 38 West Lake Avenue, ShangChen District, Hangzhou, China. ${ }^{2}$ Department of Pathology, The Third Hospital of Hangzhou, 38 West Lake Avenue, ShangChen District Hangzhou, China.

Received: 12 July 2016 Accepted: 13 August 2016

Published online: 23 August 2016

\section{References}

1. Bradley D, Ratcliffe O, Vincent C, et al. Inflorescence commitment and architecture in Arabidopsis. Science. 1997;275:80-3.

2. Hengst $U$, Albrecht $H$, Hess D, Monard D. The phosphatidylethanolaminebinding protein is the prototype of a novel family of serine protease inhibitors. J Biol Chem. 2001;276:535-40.

3. Wang X, Li N, Liu B, Sun H, Chen T, et al. A novel human phosphatidylethanolamine-binding protein resists tumor necrosis factor alpha induced apoptosis by inhibiting mitogen-activated protein kinase pathway activation and phosphatidylethanolamine externalization. J Biol Chem. 2004:279:45855-64.

4. Qiu J, Yang G, Shen Z, Xie Y, Wang L. hPEBP4 as a predictive marker for the pathological response of rectal cancer to preoperative radiotherapy. Int J Colorectal Dis. 2013;28:241-6.

5. Qiu J, Yang G, Lin A, Shen Z, Wang D, Ding L. Human phosphatidylethanolamine-binding protein 4 promoted the radioresistance of human rectal cancer by activating Akt in an ROS-dependent way. PLoS One. 2014;9(3):e90062.

6. Wang X, Li N, Li H, Liu B, Qiu J, et al. Silencing of human phosphatidylethanolamine-binding protein 4 sensitizes breast cancer cells to TNFa-induced apoptosis and cell growth arrest. Clin Cancer Res. 2005;11:7545-53.

7. Li H, Wang X, Li N, Qiu J, Zhang Y, et al. hPEBP4 resists TRAlL-induced apoptosis of human prostate cancer cells by activating Akt and deactivating ERK1/2 pathways. J Biol Chem. 2007;282:4943-50.

8. Li P, Wang X, Li N, Kong H, Guo Z, et al. Anti-apoptotic hPEBP4 silencing promotes TRAIL-induced apoptosis of human ovarian cancer cells by activating ERK and JNK pathways. Int J Mol Med. 2006;18:505-10.

9. Zhang Y, Wang X, Xiang Z, Li H, Qiu J, et al. Promotion of cellular migration and apoptosis resistance by a mouse eye-specific phosphatidylethanolamine-binding protein. Int J Mol Med. 2007;19:55-63.

10. Qiu J, Xiao J, Han C, Li N, Shen X, Jiang H, Cao X. Potentiation of tumor necrosis factor-alpha-induced tumor cell apoptosis by a small molecule inhibitor for anti-apoptotic protein hPEBP4. J Biol Chem. 2010; 285(16):12241-7.

11. Lacher MD, Tiirikainen MI, Saunier EF, Christian C, Anders M, et al. Transforming growth factor-beta receptor inhibition enhances adenoviral infectability of carcinoma cells via up-regulation of Coxsackie and Adenovirus receptor in conjunction with reversal of epithelial-mesenchymal transition. Cancer Res. 2006;66:1648-57.

12. Wildeman MA, Gibcus JH, Hauptmann M, Begg AC, van Velthuysen ML, Hoebers FJ, Mastik MF, Schuuring E, van der Wal JE, van den Brekel MW Radiotherapy in laryngeal carcinoma: can a panel of 13 markers predict response? Laryngoscope. 2009;119:316-22.

13. Khor LY, Moughan J, Al-Saleem T, Hammond EH, Venkatesan V, Rosenthal SA, Ritter MA, Sandler HM, Hanks GE, Shipley WU, Pollack A. BCl-2 and Bax expression predict prostate cancer outcome in men treated with androgen deprivation and radiotherapy on radiation therapy oncology group protocol 92-02. Clin Cancer Res. 2007:13:3585-90.

14. Saigusa S, Tanaka K, Toiyama Y, Yokoe T, Okugawa Y, Kawamoto A, Yasuda H, Morimoto $Y$, Fujikawa H, Inoue Y, Miki C, Kusunoki M. Immunohistochemical features of CD133 expression: association with resistance to chemoradiotherapy in rectal cancer. Oncol Rep. 2010;24:345-50.

15. Smith L, Qutob O, Watson MB, Beavis AW, Potts D, Welham KJ, Garimella V, Lind MJ, Drew PJ, Cawkwell L. Proteomic identification of putative biomarkers of radiotherapy resistance: a possible role for the $26 \mathrm{~S}$ proteasome? Neoplasia. 2009;11:1194-207.

16. Fu TJ, Pcng J, Lee $\mathrm{G}$, et al. Cyclin $\mathrm{K}$ functions as a CDK9 regulatory subunit and participates in RNA polymerase transcription. J Biol Chem. 1999:274:34527-30.

17. Singleton BK, Pricstlcy A, Steingrimsdottir $H$, et al. Molecular and biochemical characterization of xrs mutants defective in Ku80. Mol Ccll Biol. 1997;17:1264-73.

18. Bussing $\mathrm{CH}$, Alt FW. The cellular response to general and programmed DNA double strand breaks. DNA Repair (Amst). 2004;3:781-96.

19. Xic S, Shun XF, Shang K, et al. Relevance of LIG4 gene polymorphisms with cancer susceptibility: evidence from meta-analysis. Sci Rcp. 2014;4:6630.

20. Thackcr J, Zdzicnicka MZ. The XRCC gene: expanding role in DNA double-strand break repair. DNA Repair (Amst). 2004:3:1081-90. 